\title{
NOTES ON A LINGUISTIC DESCRIPTION AS THE BASIS FOR AUTOMATIC IMAGE UNDERSTANDING
}

\author{
RySZARd TADEUSIEWICZ*, MAREK R. OGIELA*, PIOTR S. SZCZEPANIAK **,*** \\ * Departament of Automatics \\ AGH University of Science and Technology, al. Mickiewicza 30, 30-059 Cracow, Poland \\ e-mail: \{rtad, mogiela\} @agh.edu.pl \\ ${ }^{* *}$ Institute of Computer Science \\ Technical University of Łódź, ul. Wólczanska 215, 90-924 Łódź, Poland \\ e-mail: piotr@ics.p.lodz.pl \\ *** Systems Research Institute \\ Polish Academy of Sciences, ul. Newelska 6, 01-447 Warsaw, Poland
}

\begin{abstract}
The main paradigm of image understanding and a concept for its practical machine realisation are presented. The crucial elements of the presented approach are the formalisation of human knowledge about the class of images that are to be automatically interpreted, a linguistic description and the realization of cognitive resonance.
\end{abstract}

Keywords: automatic understanding, computer vision, digital images, semantics, image processing.

\section{Introduction}

Computers can collect images, store them in databases, present them on webpages, send through the Internet, and perform many kinds of image processing operations. As a result of such operations, the form of an image can substantially change. Moreover, images in computer systems can be analyzed and recognised automatically. Therefore, a new area of computer applications has been developed, namely, computer vision. A scanner or a digital camera cannot substitute for the eyes, so the computer's "vision" needs to be understood in a broad meaning. For a long time it was believed that there was a magic formula for computer vision and its discovery seemed a matter of time. Over time, the approach has changed and it is now believed that in order to have a clear machine vision, one has to combine various elements. The transition from the mechanistic approach to the one based on a "judgment call" or an "expert opinion" is supported by the notion of image understanding. It is now almost indispensable, because while dealing with images, it is not sufficient to focus exclusively on the form. In order to recover the meaning, one needs to analyse the content. Figure 1 illustrates how complex the analysis and recognition of an image can be if one does not take into account its semantic content.
If we want to solve a simple task, i.e., to count the number of chairs in an image, it turns out that a single method that describes the image in terms of contours, components, etc. is not sufficient.

The extraction of image meaning from computationally accessible features continues to be a challenge. In image data mining, we aim at retrieving the meaning of images, in other words, we aim at automatic image understanding.

In computer science, the term automatic understanding has appeared in numerous papers, but has not been applied to images. Most papers describing automatic understanding concern the area of applications that deals with text analysis. The solutions they present, often based on semantic networks (Sieckenius de Suza, 2005; Antoniou and van Harmelen, 2008) are very interesting, but they are beyond the scope of this paper. The term automatic understanding has acquired a specific meaning, which is used, for example, in machine translation of natural languages, but this area of applications is also out of scope of this paper, dedicated to automatic understanding of images. Nevertheless, we start with presenting some information about the text understanding system, which will be useful for explaining the proper meaning of automatic understanding of images. 


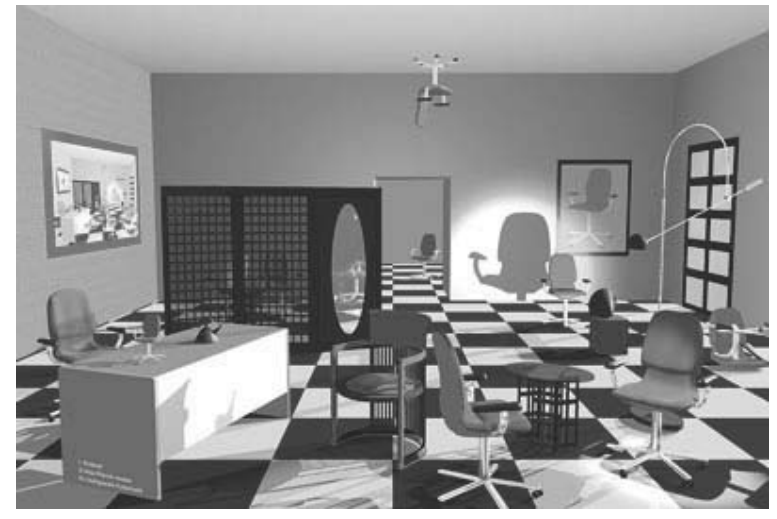

Fig. 1. Example of an image which needs the understanding of its content. Reprinted by permission from (Christensen, 2003).

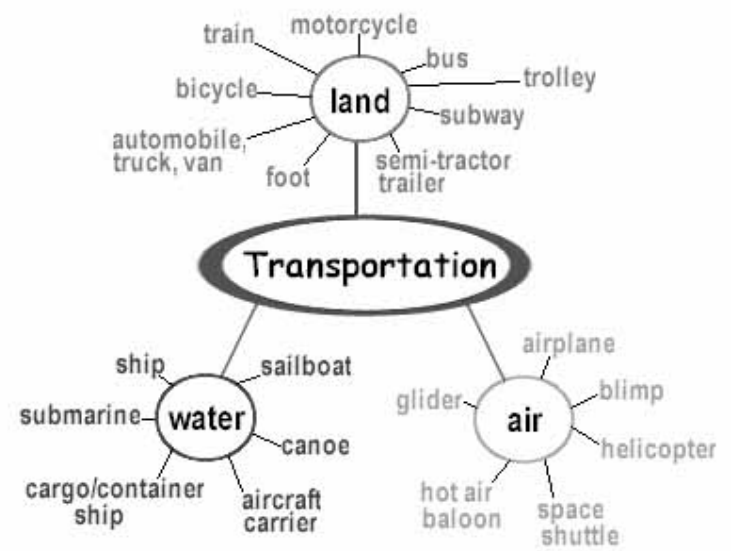

Fig. 2. Example of a simple ontology used for automatic text understanding (source: http://www.kidbibs.com/ images/semantic.gif)

The highly elaborate automatic understanding tools are used for various purposes, such as automatic retrieval of a text that is difficult to retrieve by means of key words only, automatic document summary, knowledge representation, etc. These tools are founded on ontologies, i.e., content-dependent relations between concepts. Figure 2 shows an example of a simple ontology. Numerous very good papers can be found on text content analysis including very subtle or specific domain related semantic dependencies, and this area of computer science increases and develops now very fast.

At the same time, the topic of automatic understanding of images has been addressed by relatively few authors. Homenda (2006) concentrates on a particular kind of image, namely, music notation. Leś and Tadeusiewicz (2000) advocate the analytic-geometric approach to shape understanding. The work proposes a structural (linguistic) description as the key to a successful image interpretation. Interesting results can be found in (Bowyer et al., 2008), which focuses exclusively on one kind of image,

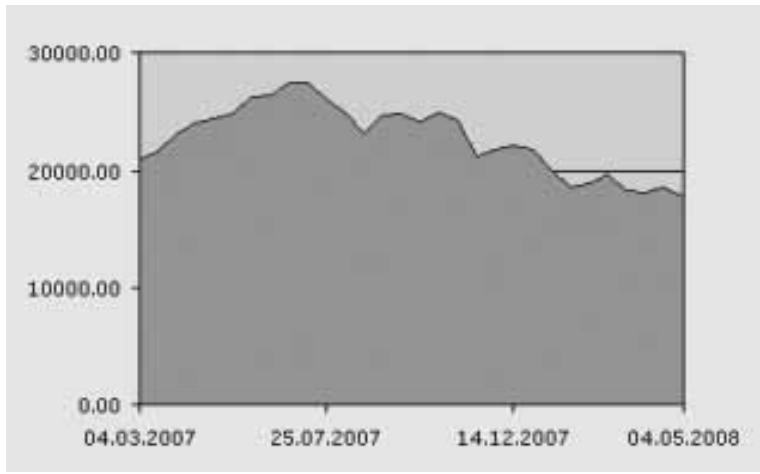

Fig. 3. Image that can be easily interpreted by an expert.

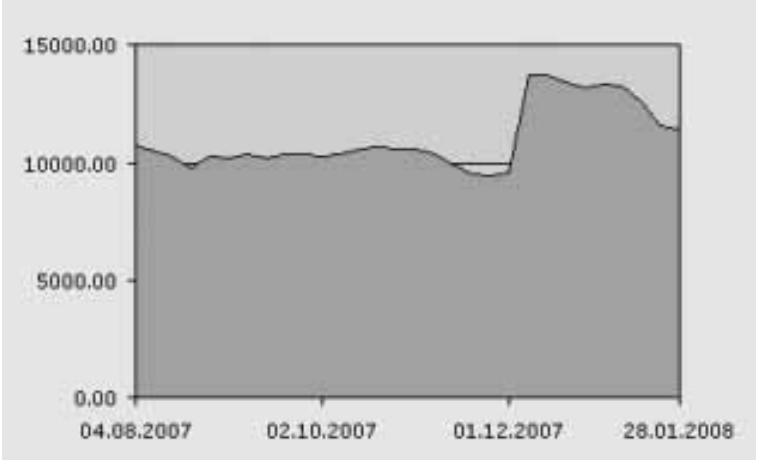

Fig. 4. Image that can help to understand a certain situation.

namely, the image of iris (for the purpose of biometric identification). Automatic understanding of people's images (mainly with the aim to identify them) is discussed in (Hilton et al., 2006). However, the approach presented in that work differs substantially from the one proposed in the paper. The idea suggested here (based on the interaction between visual data and the knowledge previously fed into the system) is related to the one found in (Drummond and Caelli, 2000; Zheng and Tsuji, 1998).

\section{Understanding of artificial images}

Understanding (even automatic) of a text is relatively easy, because every text evokes an expectation of a meaningful content. Thus, it is not unusual to consider a text in terms of meaning. Image understanding is a completely different matter. Each image has a specific form: size, colour, texture, shape, etc. It can be described in these terms and automatically recognised. However, at first glance, it may seem not to have any content, which to a large extent depends on the form of the image, the presence or absence of some objects, mutual relations between objects, etc. Given the above, can we speak of image understanding? Before we answer this question, let us discuss a few examples.

Let us consider Fig. 3 first. It shows a chart, seemingly difficult to disambiguate without additional information. However, after a careful examination of the descrip- 
tion of the axes, one can notice that the horizontal axis depicts time, whereas the vertical axis represents money (for example, euros). If we link these observations to expert knowledge about stock market trends at the turn of 2007 and 2008, we understand that the chart line shows share prices (possibly an economic slump).

Figure 4 shows a similar chart, which can be used for a more detailed understanding of a certain situation. The chart, analogously to the previous one, can be considered as a reflection of fluctuations in stock market prices. However, the phenomenon shown here is much more interesting than the one observed in the previous case. It is visible that, after a long-term stability, the prices started to rise slightly (around 6 December). This may have increased the investor's confidence and motivated him to buy securities, which resulted in a sudden rise in the line chart. Unfortunately, instead of rising, the prices started to fall and one may have lost all the money invested. Again, the meaning of the chart could be: investment mistake.

The meaning was recovered because of the interaction of two factors: the visual information from the image (chart) and the background knowledge about stock market processes. Only when the two sources of knowledge are combined can the image content be understood. The fact that the image (supported by prior knowledge) was sufficient to recover the meaning of the image indicates that an image can have a particular content, which one can try to understand. If a human can do it, a computer might be capable of doing it as well. The history of artificial intelligence (computational intelligence) shows that whenever a form of a human intellectual activity has been properly defined and described on the ground of psychology and cognitive science, it is possible to design an intelligent computer program that can imitate cognitive skills of a human being.

The analysis of images in Figs. 3 and 4 illustrates that images do have specific contents, which can be recovered thanks to proper analysis. However, critics may argue that the examples given in Figs. 3 and 4 are artificial, reflecting relatively uncomplicated events and, consequently, their meaning can be easily recovered. The next section deals with images taken from real life, which carry meanings that cannot be easily inferred from a mere form, but must be understood.

\section{Understanding of real images}

With images that represent real situations, recovering the meaning is more difficult, but not impossible. Again, let us use a few examples.

Figure 5 depicts objects that can be identified as people, a building and its elements. Looking at the image, one can say that the people are dressed in a similar way, with one identical element-the cap. Based on prior knowledge, one can infer from the photo that it might have

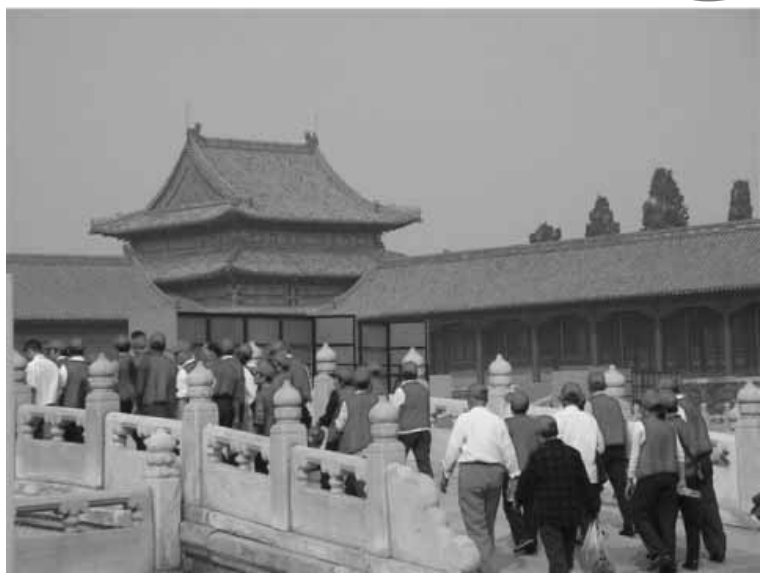

Fig. 5. Image that requires understanding, not only simple analysis.

been taken in China and that the people depicted in it are tourists, who are wearing identical clothes and caps so that they are not lost during a sightseeing trip.

Therefore, dwelling into the meaning (not focusing exclusively on the form) one can understand what the image really depicts and what subject matter it represents.

Let us consider the contents of Fig. 6. Similar objects are shown: women, men and cars. However, their meaning is different. Hence, when we search for the same message or the same story, image retrieval on the basis of object analysis can give wrong results.

The aim of this paper is to explain how computers can be made to understand, ignoring (as much as possible) the form. The answer is automatic image understanding.

\section{Proposed concept of automatic understanding}

Notice that, in order to examine the semantic content of a given message, it is necessary to combine the content with prior knowledge possessed by an intelligent agent. This applies to every message (also in the image form) and every object that aims at understanding-even a computer, whose actions result in cognitive analysis defined as automatic understanding. Figure 7 shows a general scheme of understanding. The most important element of the scheme is the cognitive matching process, which joins two streams of information. The first one is connected with an external data source. Taking the data from outside and aiming at the understanding of its merit sense, we must process the data. To be more specific, we must extract from the data the most important features. However, the process of automatic understanding is not limited to this kind of processing. If we aim at understanding data contents, we must refer to the internal resources of knowledge that are located in the mind (natural or artificial) of the agent which tries to understand the data. 

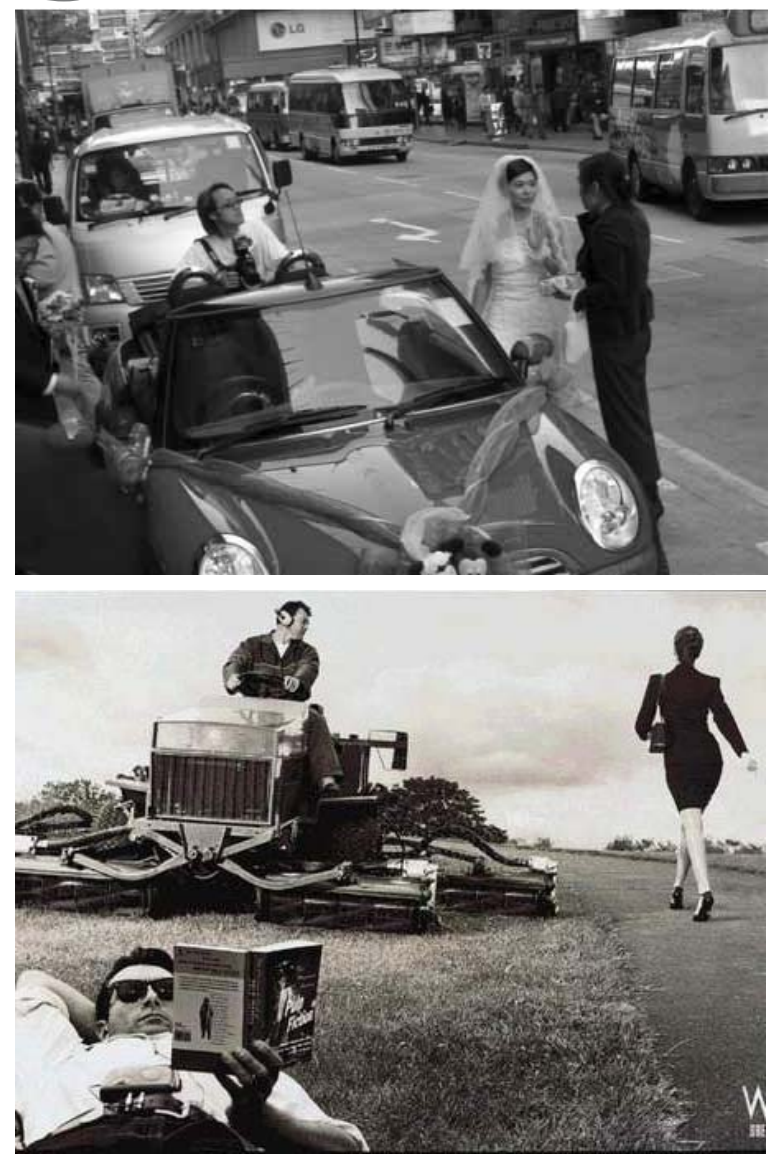

Fig. 6. Two images—-both showing women, men and vehicles.

The internal resources of knowledge are necessary for the generation of the expectations, which must be satisfied if a merit sense is to be extracted form the data. The internal resources of knowledge act as a hypothesis generator. Each hypothesis suggests a possible way of understanding, which is based on the merit knowledge located in the system. There are various types of knowledge sources. They can be coupled during the learning process, received from human experts, based on experimental anal-

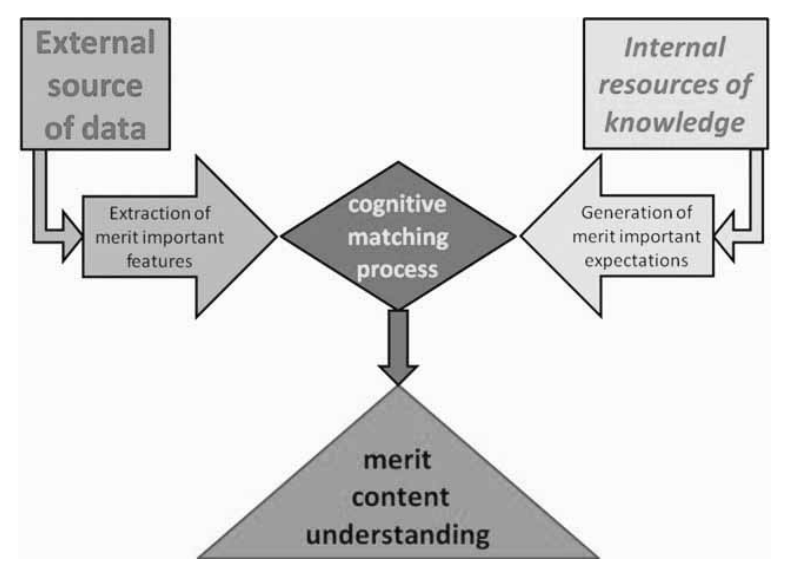

Fig. 7. General scheme of understanding. ysis of similar examples, etc. However, regardless of its type, a knowledge source can be used for describing the expectations.

The internal resources of knowledge can generate many hypotheses and expectations related to these hypotheses. When the features extracted from the input data match the expectations, a hypothesis becomes more reliable. For a proper data interpretation, we expect more than one match between the stream of data features and the expectations knowledge base. If we obtain many good matches, it means that the automatic understanding process has been successful.

\section{Linguistic description as the basis for automatic image understanding}

Having briefly discussed the notion of automatic understanding, let us try to outline the basic assumptions of the concept of automatic image understanding. Starting with the chart in Fig. 7, we shall try to adapt it to the needs of automatic image understanding. Earlier research in this domain was founded on the scheme proposed by Ogiela et al. (2008), whose work was a little more specific for automatic understanding of images (not text messages). The scheme is shown in Fig. 8. There are a few elements that need to be commented on.

First of all, when compared with Fig. 7, the input channel (camera-generated) and internal information link, which carries expectations generated by the knowledge base, are much more elaborate. As shown, the input image should be represented in a special way in the system that is supposed to understand it, and the system's actions cannot be limited to mechanical processing or routine analysis, with possible transition to automatic recognition (not to be mistaken for automatic understanding). The image which is computationally processed (with the aim of content understanding) should be represented in the system by means of linguistic methods, namely, as a chain of gram-

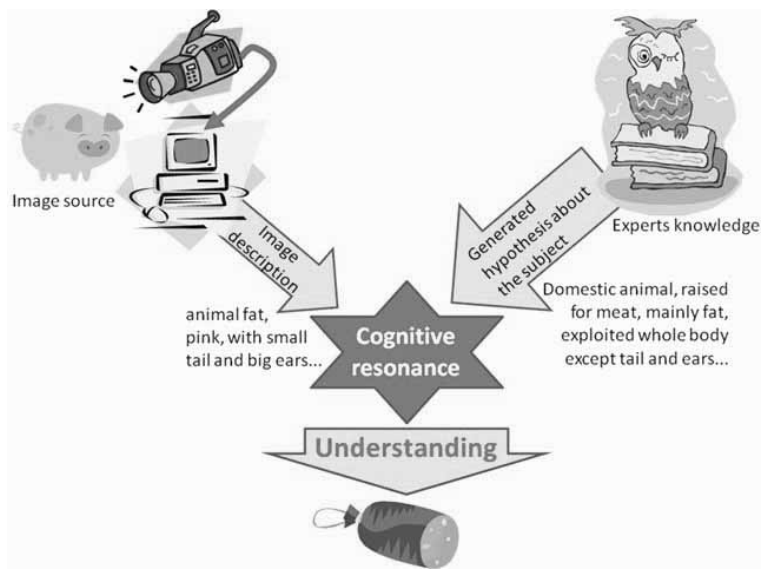

Fig. 8. Method of dealing with images that aims at automatic understanding of their contents. 


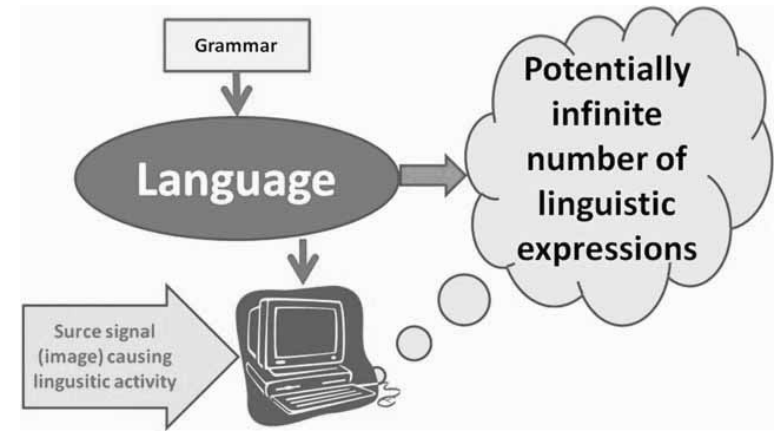

Fig. 9. Language as a tool that enables a device with limited capabilities (computer) to make an unlimited number of records.

mar terminal symbols.

Let us explain why linguistic-based tools are best for automatic image description. The reason is strictly connected with the very nature of the comprehension process, completely different from the process of recognition, which it is frequently mistaken for. When the goal is recognition, we first establish a list of categories by means of which we can classify the objects analysed. Such a classification always comprises a finite number of categories (usually including the category of an "unknown object", indicated in the picture with a question mark). The task of the algorithm that analyses the image is to determine which of the categories a given object belongs to.

Image understanding (achieved by a human, or obtained automatically) means recovering the implicit meaning of the image. The difference between recognition and understanding is that the former results in a determined set of elements (solutions provided by the system), whereas the latter is unpredictable and for this reason the set of possible image interpretations contains an infinite number of elements-allowing for the fact that computer is a device of limited capabilities, which might be a serious problem (Fig. 9).

What generates an infinite number of combinations from a finite number of elements is language. For example, the Polish language consists of a finite number of words and a finite number of grammatical rules. However, it allows one to create an infinite number of articles, novels, poems, official documents, etc. This applies also to artificial languages, such as $\mathrm{C}++$, which also consist of a definite number of components and rules. However, they are able to create an indefinite number of programs, with the possibility to constantly create new ones.

\section{Role of reasoning}

In the literature, the role of reasoning in automatic image understanding has so far been rarely considered because of its difficulty. Let us demonstrate an example. Working with the images given in Fig. 10, we can set the following tasks:
(1) recognition of English and Chinese letters,

(2) recognition of words in English and Chinese,

(3) knowing the meaning of Chinese texts in the images (a) and (c), and reason about the information given in the image (b).

Tasks 1 and 2 can be solved with the use of a classification method. The same method can be applied if there is an explanation in the database (a label) for the combination of letters given in the image (b).

But if no explaining label exists, then the computer can only state an "unknown object". As opposed to a computer, a human who knows English is able to reason about the meaning ("entrance") of information given in the image (b) in Chinese.

\section{Summary}

The approach of automatic understanding of images described in (Tadeusiewicz and Ogiela, 2004) is devoted to medical applications (Ogiela et al., 2006a; 2006b; 2006c; 2006d; Tadeusiewicz and Ogiela, 2005; Przelaskowski et $a l ., 2007)$. However, its generality allows us to extract some fundamental issues.

Trying to explain what automatic understanding is and how we can force the computer to understand the image content, we must demonstrate the fundamental difference between a formal description of an image and the content meaning of the image, which can be discovered by an intelligent entity capable of understanding the profound sense of the image in question. The fundamental features of automatic image understanding are as follows:

1. Imitation of the human way in image analysis and in reasoning about the content. An expert and a welldefined field of interest are preferred here. For example, consider medical images of some organ and a restricted class of variations in the image content.

2. Linguistic description of the image content. Examples of languages and grammars used for that purpose can be found in the book (Tadeusiewicz and Ogiela, 2004) and the papers (Tadeusiewicz et al., 2008; Ogiela and Tadeusiewicz, 2001; 2008).

3 . The image content linguistic description constructed in this manner constitutes the basis for understanding the image merit content.

The most important difference between all traditional methods of automatic image processing and the new paradigm for image understanding is that there is a feed forward data flow in the traditional methods while in the new paradigm (Tadeusiewicz and Ogiela, 2004) there are two-directional interactions between signals (features) extracted from image analysis and expectations resulting from the knowledge of the image content as given by experts. 


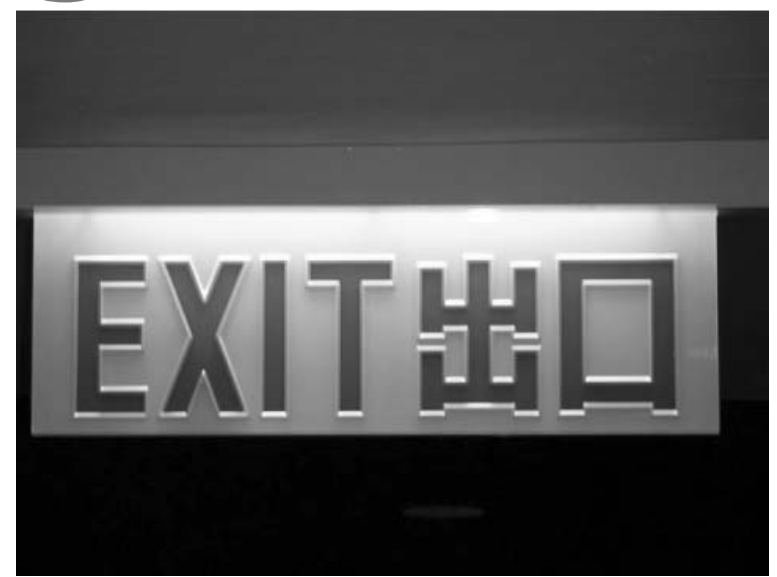

(a)

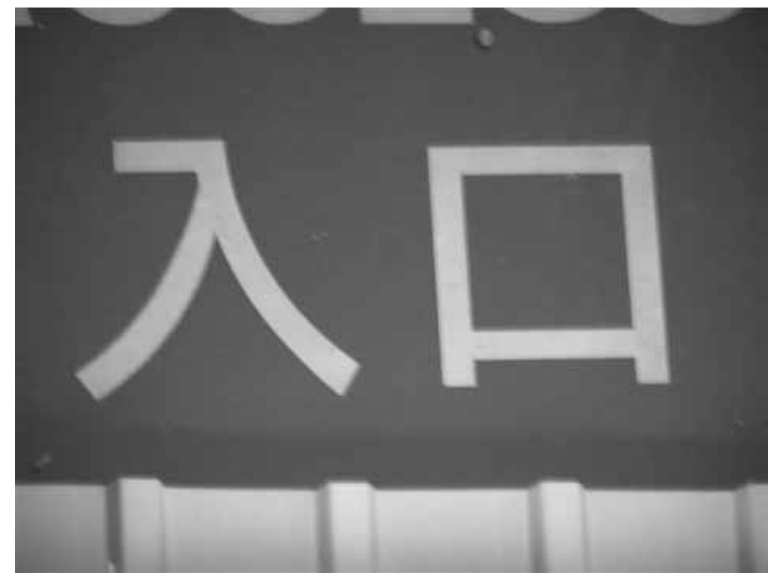

(b)

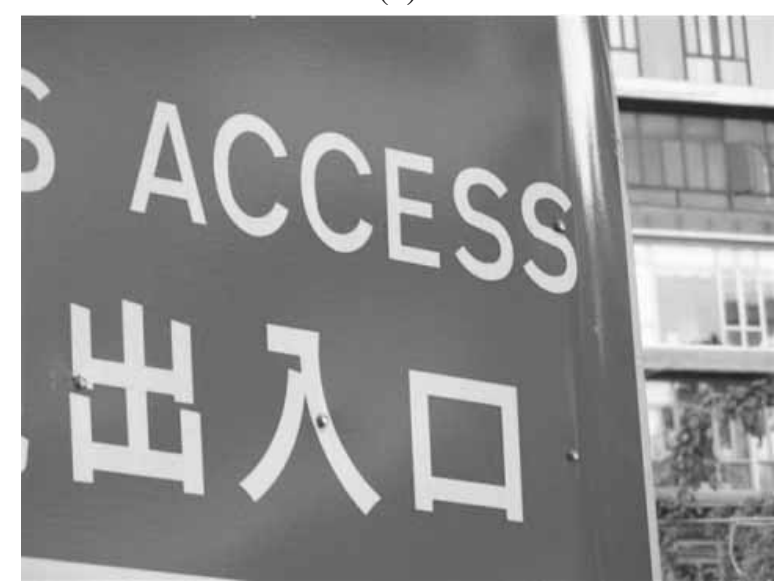

(c)

Fig. 10. Recognition, classification and understanding by reasoning: (b) EXIT, (c) ??? , (d) ACCESS.

Linguistic-based tools used for the description of images with the view of their automatic interpretation were described in (Ogiela and Tadeusiewicz, 2001; Tadeusiewicz and Ogiela, 2002) and thoroughly discussed in (Ogiela and Tadeusiewicz, 2008). Medical applications are presented in (Ogiela et al., 2006; 2008).
The idea of cognitive resonance appeared in (Ogiela and Tadeusiewicz, 2003) and was discussed in (Tadeusiewicz and Ogiela, 2004), both works being inspiring sources of knowledge for those interested in this topic.

Notwithstanding the research mentioned above, automatic image understanding technology is still in the early phase of development. One of the recent ideas is the application of adaptive potential active hypercontours (Tomczyk, 2005; Tomczyk and Szczepaniak, 2005; 2006) to image content verification (Tomczyk and Szczepaniak, 2007; 2008).

To conclude, the authors sketched here the basis for further research contributing to the development of an efficient methodology of automatic image understanding.

\section{Acknowledgment}

This work has been partly supported by the Polish Ministry of Science and Higher Education under Grant No. N 51900732/0978.

\section{References}

Antoniou, G. and Harmelen, van F. (2008). A Semantic Web Primer, MIT Press, Cambridge, MA.

Bowyer, K. W., Hollingsworth, K. and Flynn, P.J. (2008). Image understanding for iris biometrics: A survey, Computer Vision and Image Understanding 110(2): 281-307.

Christensen, H. (2003). Cognitive (Vision) Systems, ERCIM News No. 53, Special Theme: Cognitive Systems, available at http://www.ercim.org/publication/ Ercim/News/enw53/christensen.html

Drummond, T. and Caelli, T. (2000). Learning task-specific object recognition and scene understanding, Computer Vision and Image Understanding 80(3): 315-348.

Hilton, A., Fua, P. and Ronfard, R. (2006). Modeling people: Vision-based understanding of a person's shape, appearance, movement, and behaviour, Computer Vision and Image Understanding 104(2-3): 87-89.

Homenda, W. (2006). Automatic understanding of images: Integrated syntactic and semantic analysis of music notation, Proceedings of the International Joint Conference on Neural Networks, Vancouver, Canada, pp. 3026-3033.

Leś, Z. and Tadeusiewicz, R. (2000). Shape understanding system, polygon class processing methods, in $\mathrm{M}$. $\mathrm{H}$. Hamza (Ed.), Signal Processing and Communications, IASTED/ACTA Press, Anaheim, pp. 447-454.

Przelaskowski, A., Podsiadły, Marczykowska T., Wroblewska A., Boniński, P. and Bargiel, P. (2007). Computer-aided interpretation of medical images: Mammography case study, Machine Graphics \& Vision 16(3/4): 347-375

Ogiela, L., Tadeusiewicz, R. and Ogiela, M.R. (2008). Cognitive techniques in medical information systems, Computers in Biology and Medicine 38(4): 501-507. 
Ogiela, M.R. and Tadeusiewicz, R. (2001). Image understanding methods in biomedical informatics and digital imaging, Journal of Biomedical Informatics, Computers and Biomedical Research 34(6): 377-386.

Ogiela, M. R. and Tadeusiewicz, R. (2008). Modern Computational Intelligence Methods for the Interpretation of Medical Image, Springer-Verlag, Berlin.

Ogiela, M.R. and Tadeusiewicz, R. (2003). Artificial intelligence structural imaging techniques in visual pattern analysis and medical data understanding, Pattern Recognition 36(10): 2441-2452.

Ogiela, M.R., Tadeusiewicz, R. and Ogiela, L. (2006). Image languages in intelligent radiological palm diagnostics, Pattern Recognition 39(11): 2157-2165.

Ogiela, M.R., Tadeusiewicz, R. and Trzupek, M. (2008). Graphbased semantic description and information extraction in analysis of 3D coronary vessels visualizations, in $\mathrm{C}$. Badica, M. Paprzycki (Eds.), Advances in Intelligent and Distributed Computing, Springer-Verlag, Berlin, pp. 303-309.

Ogiela, L., Tadeusiewicz, R. and Ogiela, M.R. (2006a). Cognitive approach to visual data interpretation in medical information and recognition systems, in N. Zheng, X. Jiang, X. Lan (Eds.), Advances in Machine Vision, Image Processing, and Pattern Analysis, Springer-Verlag, Berlin, pp. 244-250.

Ogiela, L., Tadeusiewicz, R. and Ogiela, M.R. (2006b). Cognitive computing in intelligent medical pattern recognition systems, in D.-S. Huang, K. Li, G.W. Irwin (Eds.), Intelligent Control and Automation, Springer-Verlag, Berlin, pp. 851-856.

Ogiela, M.R., Tadeusiewicz, R. and Ogiela, L. (2006c). Graph image language techniques supporting radiological, hand image interpretations, Computer Vision and Image Understanding 103(2): 112-120.

Ogiela, M.R., Tadeusiewicz, R. and Ogiela, L. (2006d). Image languages in intelligent radiological palm diagnostics, Pattern Recognition 39(11): 2157-2165.

Sieckenius, de Suza C. (2005). The Semiotic Engineering of Human-Computer Interactions, MIT Press, Cambridge, MA.

Tadeusiewicz, R. and Ogiela, M. R. (2002). Automatic understanding of medical images-New achievements in syntactic analysis of selected medical images, Biocybernetics and Biomedical Engineering 22(4): 17-29.

Tadeusiewicz, R. and Ogiela, M.R. (2004). Medical Image Understanding Technology, Springer-Verlag, Berlin.

Tadeusiewicz, R. and Szczepaniak, P.S. (2008). Basic concepts of knowledge-based image understanding, in N.T. Nguyen, E. Puchala, M. Wozniak and A. Zolnierek (Eds.), KESAMSTA 2008, Proceedings LNAI 4953, Springer-Verlag, Berlin, pp. 42-52.

Tadeusiewicz R., Ogiela, M. R. (2005). Picture languages in automatic radiological palm interpretation, International Journal of Applied Mathematics and Computer Science 15(2): 305-312
Tadeusiewicz, R., Ogiela, L. and Ogiela, M.R. (2008). The automatic understanding approach to systems analysis and design, International Journal of Information Management 28(1): 38-48.

Tomczyk, A. (2005). Active hypercontours and contextual classification, Proceedings of the 5-th International Conference on Intelligent Systems Design and Applications ISDA'2005, Wrocław, Poland, pp. 256-261.

Tomczyk, A. and Szczepaniak, P.S. (2005). On the relationship between active contours and contextual classification, in M. Kurzyński, G. S. Jo, R. J. Howlett and L. C. Jain (Eds.), Computer Recognition Systems. Proceedings of the 5-th International Conference on Computer Recognition Systems-CORES'05, Springer, Berlin, pp. 303-310.

Tomczyk, A. and Szczepaniak, P.S. (2006). Adaptive potential active hypercontours, Proceedings of the 8-th International Conference on Artificial Intelligence and Soft ComputingICAISC'2006, Springer-Verlag, Berlin, pp. 692-701.

Tomczyk, A. and Szczepaniak, P.S. (2007). Contribution of active contour approach to image understanding, Proceedings of the IEEE International Workshop on Imaging Systems and Techniques IST'2007, Cracow, Poland, (on CDROM).

Zheng, J.Y. and Tsuji, S. (1998). Generating dynamic projection images for scene representation and understanding, Computer Vision and Image Understanding 72(3): 237-256.

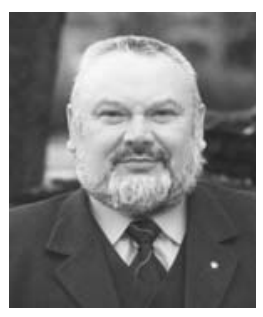

Ryszard Tadeusiewicz studied at the Electrical Engineering Department of the University of Mining and Metallurgy in Cracow (Poland), from which he graduated (with honors) in 1971 Additionally, after receiving his degree in automatic control engineering, he studied at the Faculty of Medicine at the Medical Academy in Cracow, and the undertook studies in the field of mathematical and computer methods in economics. He has written over 600 scientific papers, published in prestigious Polish and foreign scientific journals as well as numerous conference presentations-both national and international. Prof. Tadeusiewicz has also authored over 70 scientific monographs and books, including highly popular textbooks (which have had many editions). He has been the supervisor of 56 doctoral theses and a reviewer of more than 200 doctoral theses. In 2007, Polish scientists elected him a chapter chair of the Poland Section of the IEEE Computational Intelligence Society.

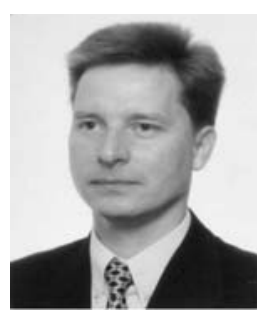

Marek R. Ogiela, Ph.D., D.Sc, works at the Bio-Cybernetics Laboratory at the AGH University of Science and Technology in Cracow. In 1992 he graduated from the Mathematics and Physics Department of Jagiellonian University. In 1996 he was granted the Ph.D. degree in control engineering and robotics by the Faculty of Electrical, Automatic Control, Computer Science and Electronic Engineering of the AGH University of Science and Technology. In 2001 he was granted the title of doctor habilitatus in computer science for his research on medical image automatic analysis and understanding. In 2005 he received the professorial title in technical sciences. He is a member of numerous world scientific associations as well as a member of the Interdisciplinary Scientific Committee of the Polish Academy of Arts 
and Sciences. He is the author of more than 130 scientific international publications on pattern recognition and image understanding, artificial intelligence, IT systems, and biocybernetics. He has been awarded many scientific honors, including Prof. Takliński's award (twice), was the first winner of Prof. Engel's award, and was nominated in the Science category for the Silver Nike award in 2003.

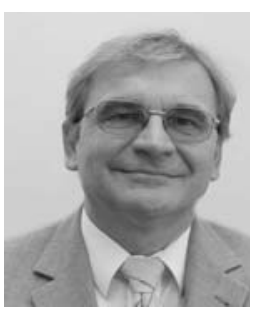

Piotr S. Szczepaniak received the M.Sc. degree in automatic control from the Faculty of Electrical Engineering of the Technical University of Łódź, Poland, in 1977, as well as the Ph.D. degree in automatic control and the D.Sc. degree (habilitation) in computer science from the Technical University of Dresden, Germany, in 1982, and 1990, respectively. He is currently a full-rank professor, having obtained the title in 2005 from the President of the Republic of

Poland. In the years 1996-2003 he was the head of the Institute of Computer Science of the Technical University of Łódź, and in 20052008 - the dean of the Faculty of Technical Physics, Computer Science and Applied Mathematics. Since 2008 he has been a deputy rector of the University. Since 2000 he has also been with the Systems Research Institute of the Polish Academy of Sciences in Warsaw. In 2001, he was appointed by the French Ministry of National Education to the position of professeur invité at Université d'Artois in Bethune, France.
His research experience covers optimal control theory, sensitivity analysis, and the approximation of dynamic time-delay systems. At present he works in the field of computational intelligence (mainly neural networks, fuzzy systems, and genetic algorithms) and its applications to modeling, simulation, image analysis, pattern recognition, knowledge extraction, intelligent Internet exploration, fault diagnosis of technical plants, and the development of medical systems. His publishing activity is evidenced by over 150 individual or collective journal papers and conference contributions, as well as by the edition of books published by Springer-Verlag. He is a member of the Polish Cybernetic Society, Polish Neural Networks Society, European Society for Fuzzy Logic and Technology, Web Intelligence Consortium, IEEE Signal Processing Society, and a Senior Member of the IEEE Computer Society. He is a coeditor-in-chief of the international Journal of Applied Computer Science (JACS) published by the Technical University of Łódź, a member of editorial boards and a guest editor of international journals, the chairman or a co-chairman of the international symposia System-Modeling-Control (1998, 2001, 2005, 2007), a co-chairman of the Atlantic Web Intelligence Conference AWIC (2003, Spain; 2005, Poland; 2006, Israel; 2007 France), a member of program committees of many conferences, and the organizer of conference sessions (e.g., 15th IMACS World Congress) He received the award of the Minister of Science, Higher Education and Technology (1993), and since 1995 he has been a Bachelor of the Silver Cross of Merit received from the President of the Republic of Poland.

Received: 15 July 2008 Revised: 29 October 2008 